\title{
Phase-field simulations of the interaction between a grain boundary and an evolving second-phase particle
}

\author{
Kunok Chang, ${ }^{\mathrm{a}, \mathrm{b}}$, Nele Moelans ${ }^{\mathrm{a}}$ \\ ${ }^{a}$ Department of Materials Engineering, KU Leuven, Kasteelpark Arenberg 44, box 2450, \\ B-3001 Heverlee, Belgium \\ ${ }^{b}$ Nuclear Materials Division, Korea Atomic Energy Research Institute (KAERI), 989-111 \\ Daedeok-daero, Yuseong-gu, Daejeon, 305-353, Korea
}

\begin{abstract}
We performed phase-field simulations to analyze the interaction of a migrating grain boundary with an evolving second-phase particle. It is found that, depending on the difference between the interfacial energies of the particle matrix interface for the 2 grain orientations involved and the driving force for grain boundary movement, particles with a particle size well above the critical limit can dissolve due to passage of the boundary.
\end{abstract}

Keywords: Phase-field modeling, Zener pinning, Particle dissolution

\section{Introduction}

Incorporating second-phase particles is one of the most efficient ways to hinder grain growth in polycrystalline materials [1-3]. Second-phase particles act as obstacles in grain boundary migration; they retard and can even stop grain growth. Because of its significance in many applications, grain growth in the presence of second-phase particles has been extensively investigated for many decades [1-6]. The pinning effect is, for example, vastly employed in the manufacturing of microalloyed steels for structural applications, where a small grain size is often required to obtain good strength, toughness and deformability [7-9].

The present understanding mainly depends on the Zener theory [1], which postulates that the second-phase particles are spherical, incoherent, monosized, and do not evolve. Therefore, a certain fraction of the grain boundary is removed when it meets a second-phase particle and has to be recreated when the grain boundary escapes from the particle. In most former computational studies on the interaction between a grain boundary and a precipitate using the phase-field method [10, 11], finite element method [12] or Monte Carlo Potts model [13, 14], the second-phase particle was therefore assumed to be entirely inert during the interaction with the grain boundary. These former studies showed that the simulation techniques capture the mechanism of grain boundary removal and subsequent recreation and give results that are in good agreement with the 
Zener theory [10]. However, the pinning effects predicted by the Zener theory and the existing simulation methodologies $[10,11]$ often do not correspond with the pinning effects seen in experimental studies [15-17].

Other mechanisms have therefore been considered to describe the interaction of a grain boundary with a second-phase particle [18]. For example, for incoherent particles it was proposed that the grain boundary can pass through or envelop the particle [19], or that the particles might dissolve or rotate when a boundary passes [20]. According to Ashby, the particle morphology and the character of the particle-matrix interface also strongly affect the pinning effect [21]. Furthermore, in reality, the interface energy and the particle size and shape can change with time [22-25], especially in the case of coherent particles which become incoherent due to passage of a grain boundary. For example, Jones et al. and Yazawa et al. observed that second-phase particles dissolved due to interaction with a grain boundary [22, 23]. However, it is extremely challenging to verify the interaction mechanism between the grain boundary and the second-phase particle in great detail because of the limited resolution of transmission electron microscopy (TEM) [20]. Therefore, in a few recent studies, a computational approach such as the phase-field method has been considered as an alternative to examine the interaction mechanism of a second-phase particle with a grain boundary. For example, the role of the morphology of the second-phase particle has been examined in 2D [26] and 3D [10] using phasefield modeling.

In this study, we formulate a phase-field model that allows for second-phase particle evolution during interaction with the grain boundary and to define different particle-matrix interface energies for the 2 grains involved. The effect of particle evolution is investigated for different ratios of the interfacial energies and a range of driving forces.

\section{Phase-field modeling of particle/grain boundary interaction.}

We introduced the model system to analyze the particle/boundary interaction in 2D as presented in Fig. 1 in reference [10], with 2 grains separated by an initially flat vertical grain boundary and one particle located in the right-sided grain (see also Figs. 1 in this paper). Two order parameters $\left(\eta_{1}, \eta_{2}\right)$ were introduced to represent the two different grain orientations. A third order parameter $\left(\eta_{3}\right)$ was used to indicate second-phase particle region. An additional conserved order parameter $(c(\mathbf{r}, \mathrm{t}))$ was introduced to indicate the concentration of solute. The snapshot of the initial configuration is visualized in Fig. 1. At the very begining, even a diffused interface is not generated, we performed only 100 time steps simulations to make the interface diffuse. The interfacial energy in Fig. 1 is isotropic. Since the driving force of the grain boundary is zero, the location of the grain boundary is same as the initial position.

The Ginzburg-Landau time dependent equation

$$
\frac{\partial \eta_{i}(\mathbf{r}, t)}{\partial t}=-L \frac{\delta F}{\delta \eta_{i}(\mathbf{r}, t)}(i=1,2,3)
$$




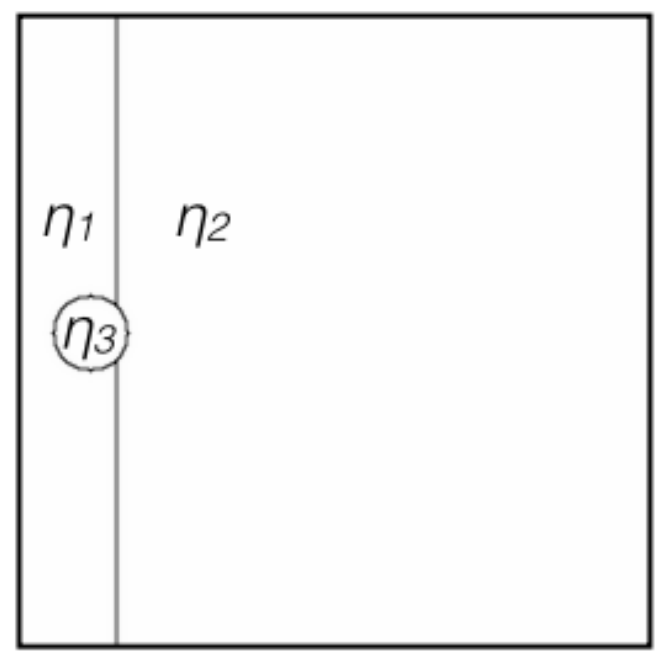

Figure 1: The snapshots of the particle-grain boundary interactions when the interfacial energy ratio is $R=1.0$ with no driving force $(\beta=0.0) \cdot \eta_{1}$ and $\eta_{2}$ indicate two different grain orientations of the matrix and $\eta_{3}$ indicates the second-phase particle. The compositions of the solute are 0.0 and 1.0 in the matrix and the particle, respectively.

was solved with respect to $\eta_{1}, \eta_{2}$ and $\eta_{3}$.

The Cahn-Hilliard diffusion equation was solved with respect to $c(\mathbf{r}, \mathrm{t})$

$$
\frac{\partial c(\mathbf{r}, t)}{\partial t}=\nabla M \nabla\left(\frac{\delta F}{\delta c(\mathbf{r}, t)}\right)
$$

where $L$ is related to the grain boundary mobility, $M$ denotes solute (interdiffusion) mobility and $F$ is the total free energy of the system. The free energy is a function of the order parameters and their gradients $[10,11]$ :

$$
F=\int_{V}\left[f_{0}\left(\eta_{1}, \eta_{2}, \eta_{3}, c\right)+\frac{1}{2} \sum_{i=1}^{3} \kappa_{i}\left(\nabla \eta_{i}\right)^{2}+\frac{1}{2} \kappa_{c}(\nabla c)^{2}\right] d V
$$

where,

$f_{0}\left(\eta_{1}, \eta_{2}, \eta_{3}, c\right)=\sum_{i=1}^{3}\left(-\frac{1}{2} \eta_{i}^{2}+\frac{1}{4} \eta_{i}^{4}\right)+\gamma_{i, j} \sum_{i=1}^{3} \sum_{i \neq j}^{3} \eta_{i}^{2} \eta_{j}^{2}+\beta H\left(\eta_{2}\right)+\phi_{m} f_{m}+\phi_{p} f_{p}$

The $\beta H\left(\eta_{2}\right)=\beta\left(-2 \eta_{2}^{3}+3 \eta_{2}^{2}\right)$ term introduces a driving force to migrate the flat grain boundary, as already used in reference [27]. $H\left(\eta_{2}\right)$ is defined such that $H(0)=0, H(1)=1$ and $\partial H / \partial \eta_{2}=0$ when $\eta_{2}=0,1 . \beta$ is a phenomenological constant that controls the magnitude of the driving force. $f_{m}, f_{p}$ are the local fraction of matrix phase and particle phase, respectively, and defined as follows: 


$$
f_{m}=\frac{\eta_{1}^{4}+\eta_{2}^{4}}{\eta_{1}^{4}+\eta_{2}^{4}+\eta_{3}^{4}}, f_{p}=1-f_{m}
$$

and,

$$
\phi_{m}=A_{m}(c-0.1)^{2}, \phi_{p}=A_{p}(c-1.0)^{2}
$$

with $A_{m}=0.5$ and $A_{p}=5.0$ in our simulations, are the composition dependent chemical free energies of the matrix and precipitate phase, respectively. As shown in Eq. 6, the equilibrium compositions of the matrix and particle are 0.1 and 1.0 , respectively.

Eq. 1 was solved using a forward Euler discretization scheme [10, 28] and Eq. 2 using the semi-implicit Fourier spectral method [29]. The parameter values $\kappa_{i}=1.0, \kappa_{c}=1.0, \Delta t=0.001, \Delta x=1.0, M=1$ and $L=1$ were set. The simulation cell was $256 \times 256$ voxels. For the non-conserved order parameters, a periodic boundary condition was applied along the $\mathrm{x}$-direction only and the condition that $\eta(y=0)=\eta(y=1)$ and $\eta(y=257)=\eta(y=256)$ was applied along the y-direction. The interfacial width and the grain boundary width are approximately three and four grid points, respectively.

The different interfacial energies were implemented through the $\gamma_{i, j}$ coefficients. $\gamma_{1,2}=1.0$ was taken to set the grain boundary energy $\sigma_{g b}$ equal to 0.605 . The $\gamma_{13}$ and $\gamma_{23}$ were set to obtain the interfacial energies $\sigma_{1}$ (interfacial energy between grain 1 and the particle) and $\sigma_{2}$ (interfacial energy between grain 2 and the particle) of the particle matrix interface for the 2 grain orientations as given in table 1 for the 3 configurations considered in this study. We defined $\sigma_{1}$ as the interfacial energy between the left grain and the second-phase particle and $\sigma_{2}$ as the interfacial energy between the right grain and the second-phase particle. Various values of $\beta$ were used to study the effect of the driving force. The initial particle diameter was 30 grid points, which is larger than the critical particle diameter for all considered particle-matrix interfacial energies. We put a 30 grid points diameter particle on the single crystal and perforemd the simulations to examine the particle stability.

\begin{tabular}{c|c|c||c|c}
$\sigma_{1}$ & $\sigma_{2}$ & $R=\sigma_{1} / \sigma_{2}$ & $\gamma_{i j}=\gamma_{1}$ & $\gamma_{i j}=\gamma_{2}$ \\
\hline 0.6919 & 1.0200 & 0.678 & 1.8 & 0.6 \\
0.7065 & 0.8932 & 0.791 & 1.4 & 0.7 \\
0.7560 & 0.7560 & 1.000 & 1.0 & 1.0 \\
0.8932 & 0.7065 & 1.264 & 0.7 & 1.4 \\
1.5120 & 0.7012 & 2.156 & 0.5 & 1.5
\end{tabular}

Table 1: Interfacial energies $\sigma_{1}$ and $\sigma_{2}$, their ratios $R=\sigma_{1} / \sigma_{2}$ and the associated model parameter values $\gamma_{1,3}$ and $\gamma_{2,3}$ used in this study. 


\section{Results and discussion}

Simulations were performed for different values of the interfacial energy ratio $R=\sigma_{1} / \sigma_{2}$ and different values of the driving force for grain boundary migration. It is always assumed that grain 1 extends into grain 2 . Initially the particle is circular. However its shape may change in the simulations.

For the larger $R$-values, namely for $R \geq 0.791$, we found that, in agreement with the assumptions in Zener's theory, there is a critical driving force for which the grain boundary can pass by the particle for larger values of the driving force and is pinned by the particle for smaller values of the driving force. This is illustrated in Figure 2 for $R=2.156$.

However, for the smallest $R$-value we tested, namely $R=0.678$, we found that there is an intermediate regime of driving forces, for which the particle dissolves due to interaction with the grain boundary, as shown in Figure 3,although the initial particle size is well above the critical stable particle size. As explained further, we believe the dissolution is a consequence of the morphological changes the particle undergoes during the particle-grain boundary interaction. Namely, to satisfy mechanical equilibrium at the junctions where the grain boundary intersects with the particle-matrix interfaces, the particle has to adapt its shape continuously while the grain boundary is passing by, which can result in dissolution of the particle. If the driving force for grain boundary movement is high, the duration of the grain boundary-particle interaction is short and there is not enough time for the particle to fully dissolve. Therefore, the particle, although shrinked, is still present in the third snapshot of Fig. 3(c). For intermediate driving forces, the interaction time becomes longer, and there is enough time for the particle to change its morphology and shrink completely during the interaction, which is the case in Fig. 3(b). When the driving force is very low, grain boundary motion is stopped before a configuration is reached for which (complete) particle dissolution occurs, as is the case in Fig. 3(c).

According to Ref. [5], Nes et al. derived the force balance equation for the case where $\sigma_{1}$ and $\sigma_{2}$ are different. They assumed the particle is spherical (circular in 2D) and does not evolve. Nes et al. derived the following relation

$$
\begin{gathered}
\sigma_{1}=\sigma_{2}+\sigma_{g b} \cos \alpha \\
\cos \alpha=\frac{\sigma_{1}-\sigma_{2}}{\sigma_{g b}}
\end{gathered}
$$

with $\alpha$ as defined in Fig. 4(a) and where $\sigma_{1}$ and $\sigma_{2}$ indicate interfacial energy between grain 1 and the particle and between grain 2 and the particle, respectively. $\sigma_{g b}$ denotes the grain boundary energy.

From Eq. 8, we derive that $\alpha>\pi / 2$ when $\sigma_{1}<\sigma_{2}$ and $\alpha \geq \pi / 2$ when $\sigma_{1} \geq \sigma_{2}$. These three situations are illustrated for a fixed circular particle in Figure 3a. Also in our simulations we have $\alpha>\pi / 2$ for the case where $R=\sigma_{1} / \sigma_{2}<1$ (see Figure 3) and $\alpha<\pi / 2$ for $R>1$ (see Figure 2).

However, in the simulations, the shape of the particle will tend to evolve from a circle to one that fulfills mechanical equilibrium in the triple junctions 


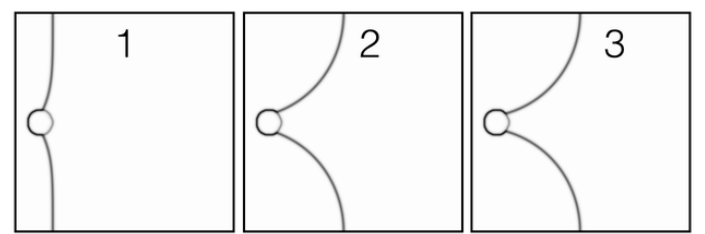

(a) $\beta=0.3200$

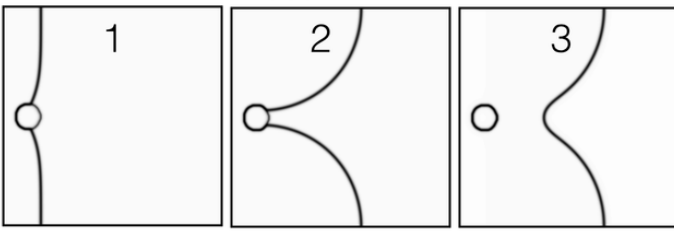

(b) $\beta=0.3300$

Figure 2: The snapshots of the particle-grain boundary interactions when the interfacial energy ratio is $R=2.156$ at three different times steps for two different driving forces ( $\beta=$ $0.3200,0.3300$ ). The steady state is described at the third snapshot of figure (a).

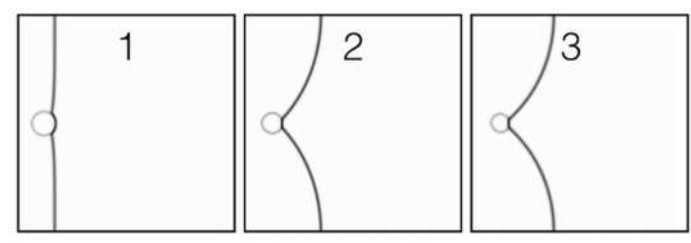

(a) $\beta=0.2372$

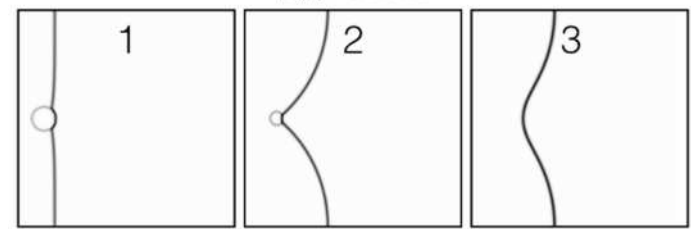

(b) $\beta=0.2374$

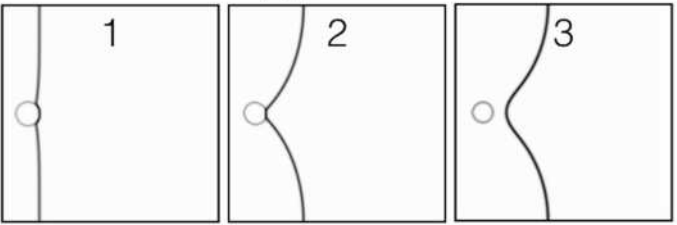

(c) $\beta=0.3000$

Figure 3: The snapshots of the particle-grain boundary interactions when the interfacial energy ratio is $R=0.678$ at three different times steps for three different driving forces $(\beta=0.2372,0.2374,0.3000)$. The steady state is described at the third snapshot of figure (a). 


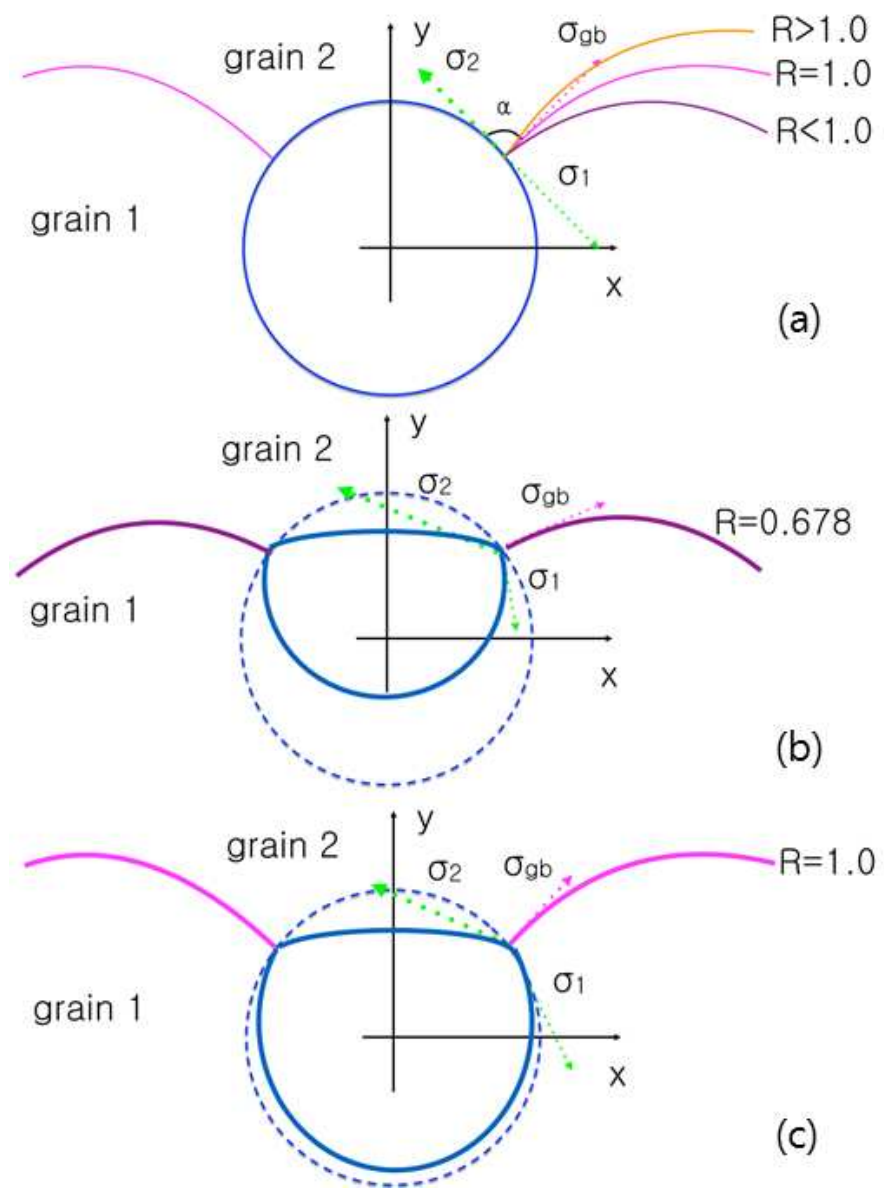

Figure 4: Shematic drawing of the interaction between a second-phase particle and the grain bounday for a) different $R$ values, assuming the particle shape and size is fixed, b) an evolving particle with $R=0.678$, and c) an evolving particle with $R=1$. 
where the grain boundary meets the particle-matrix interface. The case with $R=0.678$ is illustrated in figure $4 \mathrm{~b}$. In this case, $\sigma_{g b} \approx \sigma_{1}$ and $\sigma_{2} \gg \sigma_{1}$. Once the grain boundary is half-way the particle, the interface between the right-sided grain (grain2) and the particle then becomes flat, while the curvature of the interface between the left-sided grain (grain 1) and the particle has to increase continuously when the grain boundary moves further along the particle. This requires dissolution of the particle as seen in the simulations for intermediate driving forces. The solute diffuses and finally reprecipitates on the right side of the particle. However, as long as the grain boundary is moving through the particle, the dissolution on the left side of the particle continuous.

The dissolution of the particle is diffusion controlled and takes thus time. When the driving force for grain boundary migration is large, the grain boundary is only for a short time in contact with the particle and there is not enough time for the particle to dissolve completely (or until its size is below the critical limit for thermodynamic stability). For driving forces slightly above the maximum pinning force (the intermediate driving forces), the interaction time is maximized, so that there is enough time for complete particle dissolution or dissolution till a particle size that is below the critical particle size for thermodynamic stability.

When the driving force for grain boundary migration is small, the grain boundary stops moving before a configuration is reached for which considerable particle dissolution is required.

Also for larger $R$-values, the interface between the right-sided grain and the particle flattens, while the curvature of the interface between the left-sided grain increases; the effect, and hence the driving force for particle dissolution, is however less, as is illustrated for $R=1$ in Fig. 4c. For theses conditions, we did not see a regime at intermediate driving forces for which particle dissolution happens.

In all cases where the remaining particle size is still larger than the critical particle size after the grain boundary pinches of the particle, the particle becomes circular and the particle size increases again after full passage of the boundary.

From the set of 69 simulations performed in this study, a grain boundary/particle interaction mode map was constructed as shown in Fig. 5. For $R>1$, the average particle-matrix interfacial energy increases while the grain boundary proceeds. Thus the pinned state becomes energetically more favorable. The mode map shows indeed that the maximum pinning force increaeses as $R$ increases. As mentioned above, in our simulations, the particle dissolution only takes place for $R=0.678$ and for intermediate driving forces $(0.002374 \leq \beta \leq 0.003000)$.

It is worthy to note that when $0.002374 \leq \beta \leq 0.002600$, the particle dissolves completely during the particle/boundary interaction. For a driving force $0.002700 \leq \beta \leq 0.003000$, the complete particle dissolution occures only after the partice/boundary interaction. In this case, the particle has shrinked during the interaction to a size that is below the critical size for thermodynamic stability. 


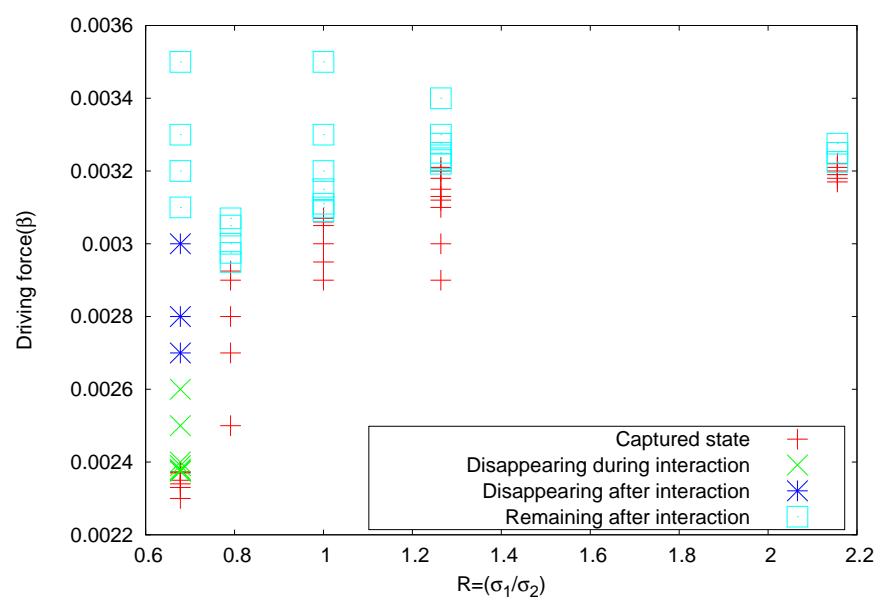

Figure 5: The grain boundary/particle interaction map as a function of the interfacial energy anisotropy $(R)$ and the driving force for grain boundary movement $(\beta)$ derived from a set of 69 phase field simulations.

\section{Conclusion}

We performed phase-field simulations of the interaction of a moving grain boundary with a second-phase particle using a model that can describe particle evolution during the particle-grain boundary interaction. We found that the morphological changes of the particle during the grain boundary-particle interaction can cause particle dissolution even for particle sizes well above the stable particle size. A grain boundary particle interaction map, considering the driving force and the interfacial energy ratio $R=\sigma_{1} / \sigma_{2}$, was derived based on the phase field simulations. It shows that this alternative mechanism becomes active for $R \leq 0.678$ and allows the grain boundary to escape from the particle at lower driving forces than if particle dissolution would not occur. It still remains to be verified if this effect is also present in 3-d, since the particle-grain boundary interaction is different in 2-d and 3-d [11]. Furthermore it would be very interesting to study the effect of particle size and solute diffusivity in the matrix on the grain boundary interaction map.

Acknowledgements: We sincerely appreciate the CREA-financing of KU Leuven, grant CREA/12/012 on Phase field modeling of the morphological evolution during phase transitions in organic materials. Parallel simulations were run on the High Performance Computer cluster of VSC (Vlaams Supercomputer Centrum). K Chang was also supported by the National Research Foundation of Korea (NRF) grant funded by the Korea government(MSIP) (No.2012M2A8A4025886). 


\section{References}

[1] C. Smith, Trans. Metall. Soc. A.I.M.E. Eng. 175 (1948) p.15.

[2] P. Hellman and M. Hillert, Scand. J. Metall. 211 (1975) p.211.

[3] M. Hillert, Acta Metall. 36 (1988) p.3177.

[4] H. Song and R.J. Coble, J. Am. Ceram. Soc. 73 (1990) p.2086.

[5] E. Nes, N. Ryum and O. Hunderi, Acta Metall. 33 (1985) p.11.

[6] F.J. Humphreys and M.G. Ardakani, Acta Mater. 44 (1996) p.2717.

[7] M. Guo and H. Suito, ISIJ Int. 39 (1999) p.1289.

[8] M. Guo and H. Suito, ISIJ Int. 39 (1999) p.1297.

[9] M. Chapa, S.F. Medina, V. López and B. Fernández, ISIJ Int. 42 (2002) p.1288.

[10] K. Chang and L.Q. Chen, Modelling Simul. Mater. Sci. Eng. 20 (2012) p.055004.

[11] N. Moelans, B. Blanpain and P. Wollants, Acta Mater. 53 (2005) p.1771.

[12] B. Kim and T. Kishi, Acta Mater. 47 (1999) p.2293.

[13] J. Gao and R.G.Thompson and B.R. Patterson, Acta Mater. 45 (1997) p.3653.

[14] M. Anderson, G. Grest, R. Doherty, K. Li and D. Srolovitz, Scripta Metall. 23 (1989) p.753.

[15] A.R. Jones and N. Hansen, Acta Metall. 29 (1989) p.589.

[16] K. Okada, Y. Yoshizawa and T. Sakuma, J. Am. Ceram. Soc. 74 (1991) p.2820.

[17] J. Pérez-Rigueiro, J.Y. Pastor, J. Llorca, M. Elices, P. Miranzo P and J.S. Moya, Acta Mater. 46 (1998) p.5399.

[18] R.D. Doherty, Metal. Science 16 (1982) p.1.

[19] P.R. Rios, Acta Metall. 35 (1987) p.2805.

[20] S.P. Ringer, W.B. Li and K.E. Easterling, Acta Metall. 40 (1992) p.275.

[21] M.F. Ashby and L. Johnson, Phil. Mag. 20 (1969) p.1009.

[22] A.R. Jones and B. Ralph, Acta Metall. 23 (1975) p.355.

[23] Y. Yazawa, T. Furuhara, T Maki, Acta Mater. 52 (2004) p.3727. 
[24] A.R. Jones, P.R. Howell and B. Ralph, J Mat. Sci. 11 (1976) p.1593.

[25] R.Z. Valiev, R.S. Musalimov, N.K. Tsenev, Phys. Stat. Sol. 115 (1989) p.451.

[26] K. Chang, W. Feng, L.Q. Chen, Acta Mater. 57 (2009) p.5229.

[27] B. Saswata, T.W. Heo, K. Chang and L.Q. Chen, Modelling Simul. Mater. Sci. Eng. 19 (2011) p.035002.

[28] C.E. Krill III and L.Q. Chen, Acta Mater. 50 (2002) p.3057.

[29] J.Z. Zhu, L.Q. Chen, J. Shen and V. Tikare, Phys. Rev. E 60 (1999) p.3564. 